\title{
The Asymptotic Expansion of the Statistical Distribution of N. V. Smirnor
}

By

\section{H. A. LAUWERIER}

\section{Introduction}

Let $F(\lambda)$ be a continuous distribution function of the observable stochastic variable $\lambda$ and let $F_{n}(\lambda)$ be the corresponding empirical distribution function which is obtained after $n$ independent observations. Surravov [1] studied the stochastic variable

$$
D_{n}^{+}=\sqrt{n} \sup _{x}\left(F_{n}^{3}-F^{\prime}\right)
$$

and showed that for $0<\lambda<\sqrt{n}$

$$
\Phi_{n}^{+}(\lambda)=P\left\{D_{n}^{+}<\lambda\right\}=1-\frac{\alpha n !}{n^{n}} \sum_{k>\alpha}^{n} \frac{(k-\alpha)^{k}(n-k+\alpha)^{n-k-1}}{k !(n-k) !}
$$

where $\alpha=\lambda \sqrt{n}$.

He also showed that for $n \rightarrow \infty$

$$
\Phi_{n}^{+}(\lambda)=1-e^{-2 \lambda^{2}}\left\{1-\frac{2 \lambda}{3 \sqrt{n}}+O\left(\frac{1}{n}\right)\right\}
$$

with $\lambda=O\left(n^{1 / 6}\right)$.

In a recent paper Chan Li Tsian [2] was able to extend the expansion (1.3) by two more terms. The fact that both authors used rather complicated methods prompted us to find the asymptotic expansion of $\Phi_{n}^{+}(\lambda)$ by other means.

Our method is based upon the following lemma, the proof of which is obvious.

Lemma 1. Let the functions $\varphi(z, \alpha), \psi(z, \alpha)$ of the complex variable $z$ and the positive real parameter $\alpha$ be determined by

$$
\begin{aligned}
& \varphi(z, \alpha)=\alpha \sum_{k=0}^{\infty} \frac{(k+\alpha)^{k-1}}{k !} z^{k}, \quad|z|<e^{-1}, \\
& \psi(z, \alpha)=\sum_{k>\alpha}^{\infty} \frac{(k-\alpha)^{k}}{k !} z^{k}, \quad|z|<e^{-1}
\end{aligned}
$$

then

$$
\varphi(z, \alpha) \psi(z, \alpha)=\sum_{n>\alpha}^{\infty} S_{n}(\alpha) z^{n}
$$

where

$$
S_{n}(\alpha)=\frac{n^{n}}{n !}\left\{1-\Phi_{n}^{+}(\lambda)\right\}
$$

Hence the asymptotic behaviour of $\Phi_{n}^{+}(\lambda)$ is essentially that of the $n^{\text {th }}$ coef- 
ficient of the power series expansion of $\varphi \psi$. Using the well-known coefficient formula

$$
S_{n}(\alpha)=\frac{1}{2 \pi i} \oint \frac{\varphi(z, \alpha) \psi(z, \alpha)}{z^{n+1}} d z,
$$

the required expansion follows easily by standard technique from the analytic behaviour of $\varphi$ and $\psi$ at the singularity which is nearest to the origin. It will be shown in the next section that this is $z=e^{-1}$ where $\phi$ and $\psi$ both have a branch point of order two.

The discussion of the analytic properties of $\varphi$ and $\psi$ involves a detailed analysis of the complex transformation $z=w \exp -w$. This will be carried out in section 2. The asymptotic expansion of $\Phi_{n}^{+}(\lambda)$ will then be derived in section 3 . Finally in the Appendix a slightly simpler derivation of the leading term of this asymptotic expansion will be given.

The reader who is interested in the method rather than in details may now proceed at once to the Appendix for which he only needs to consult the first few paragraphs of sections 2 and 3 .

Our main result may be stated as follows.

Theorem.

$$
\Phi_{n}^{+}(\lambda)=1-\frac{n !}{n^{n} e^{-n} V^{2 \pi n}} e^{-2 \lambda^{2}} \sum_{j=0} f_{j}(\mathrm{He}) n^{-1 / 2 j},
$$

where the symbolic expression $f_{j}(\mathrm{He})$ stands for a polynomial $f_{j}(t)$ in which the powers $t^{m}$ have been replaced by the Hermite polynomials $\mathrm{He}_{m}(2 \lambda)$. The polynomials $f_{j}$ are determined by the generating series

$$
A(p) \exp \left(u^{-2} B(p)\right)=\sum_{j=0}^{\infty} f_{j}\left(\frac{p}{u}\right) u^{j}
$$

where

$$
A(p)=\frac{e^{2 p}-1-2 p}{p\left(e^{2 p}-1\right)}, \quad B(p)=1+\frac{1}{2} p^{2}-p \operatorname{cth} p-\ln \frac{\operatorname{sh} p}{p} .
$$

Numerical illustration.

$$
\begin{aligned}
& A(p)=1-\frac{1}{2} p+\frac{1}{45} p^{3}-\frac{2}{945} p^{5}+O\left(p^{7}\right) . \\
& B(p)=-\frac{1}{36} p^{4}-\frac{1}{405} p^{6}+O\left(p^{8}\right) . \\
& f_{0}(t)=1, \quad f_{1}(t)=-\frac{1}{3} t, \quad f_{2}(t)=-\frac{1}{36} t^{4} \\
& f_{3}(t)=\frac{1}{45} t^{3}+\frac{1}{108} t^{5}, \quad f_{4}(t)=\frac{1}{405} t^{6}+\frac{1}{2592} t^{8}, \ldots
\end{aligned}
$$

Corollary.

$$
\begin{aligned}
e^{2 \lambda^{2}}\{1 & \left.-\Phi_{n}^{+}(\lambda)\right\}=1-\frac{\mathrm{He}_{1}(2 \lambda)}{3 \sqrt{n}}+\frac{3-\mathrm{He}_{4}(2 \lambda)}{36 n}- \\
& -\frac{15 \mathrm{He}_{1}(2 \lambda)-12 \mathrm{He}_{3}(2 \lambda)-5 \mathrm{He}_{4}(2 \lambda)}{540 n \sqrt{n}}+ \\
& +\frac{45-60 \mathrm{He}_{4}(2 \lambda)+32 \mathrm{He}_{6}(2 \lambda)+5 \mathrm{He}_{8}(2 \lambda)}{12960 n^{2}}+\cdots
\end{aligned}
$$




\section{The transformation $z=w \exp -w$}

The function $x=u \exp -u$ has for positive real $u$ the two inverses $u=f(x)$ and $u=g(x)$ with

$$
0<f(x) \leqq 1 \leqq g(x) \text { for } 0<x<e^{-1} .
$$

We have the classical result due to EIsEnstern *

$$
f(x)=\sum_{n=1}^{\infty} \frac{n^{n-1}}{n !} x^{n}
$$

where the right-hand side converges for $|x|<e^{-1}$. This formula may easily be proved by applying the complex coefficient formula in the following way

$$
\frac{1}{2 \pi i} \oint \frac{f(z)}{z^{n+1}} d z=\frac{1}{2 \pi i} \oint \frac{e^{n w}(1-w)}{w^{n}} d w=\frac{n^{n-1}}{n !},
$$

where we have used the substitution

$$
z=w \exp -w, \quad w d z / d w=z(1-w) .
$$

It follows that $w \exp -w$ has an inverse $w=f(z)$ which is holomorphic in the interior of the circle $|z|=e^{-1}$. However, the inverse $w=g(z)$ is highly singular at the origin. According to (2.3) $f(z)$ and $g(z)$ have a singularity at $z=e^{-1}$ where they have the common value 1 . A simple calculation shows that this singularity is a branch point of the order two and that in the vicinity of this point

$$
\begin{aligned}
& f(z)=1-(2-2 e z)^{1 / 2}+\frac{1}{3}(2-2 e z)+O(2-2 e z)^{3 / 2}, \\
& g(z)=1+(2-2 e z)^{1 / 2}+\frac{1}{3}(2-2 e z)+O(2-2 e z)^{3 / 2} .
\end{aligned}
$$

It will be found convenient to consider $p=1 / 2(g-f)$ as a new independent variable instead of $z$ or rather $\sqrt{2-2 e z}$. The consequences of this are collected in the following lemma the contents of which are adaptations of well-known results of classical analysis.

Lemma 2. The substitution

$$
z=\frac{p}{\operatorname{sh} p} \exp (-p \operatorname{cth} p)
$$

gives

$$
\begin{gathered}
g(z)-f(z)=2 p \\
g(z)=p e^{p} / \operatorname{sh} p, \quad f(z)=p e^{-p} / \operatorname{sh} p, \\
-\ln e z=\sum_{k=1}^{\infty} \frac{(2 k+1) B_{2 k}}{2 k(2 k) !}(2 p)^{2 k}, \\
\frac{d}{d p} \ln g(z)=1-\sum_{k=1}^{\infty} \frac{B_{2 k}}{(2 k) !}(2 p)^{2 k-1},
\end{gathered}
$$

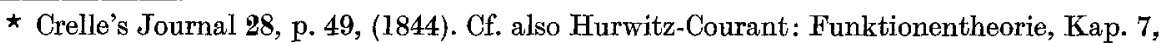
$\S 2$, Beispiel 1, and E. M. Wright: Solution of the equation $\mathrm{ze}^{z}=\mathrm{a}$, Bull. Am. math. Soc. 65, $89-93(1959)$. 
where the $B_{2 k}$ are the Bernoulli numbers

$$
B_{2}=\frac{1}{6}, \quad B_{4}=-\frac{1}{30}, \quad B_{6}=\frac{1}{42}, \quad B_{8}=-\frac{1}{30}, \ldots
$$

Numerically.

$$
\begin{aligned}
& -\ln e z=\frac{1}{2} p^{2}-\frac{1}{36} p^{4}+\frac{1}{405} p^{6}-\cdots \\
& \frac{d}{d p} \ln g(z)=1-\frac{1}{3} p+\frac{1}{45} p^{3}-\frac{2}{945} p^{5}+\cdots .
\end{aligned}
$$

We consider now the full conformal map $z=w \exp -w$.

The figure on the page 66 shows that the $z$-plane is mapped upon an infinite number of regions of the $w$-plane. These regions are separated by the curves

$$
u=v \operatorname{cotg} v .
$$

If they are numbered by I, II, etc. as shown in the figure we see that the region I corresponds to the branch $f(z)$ and II to the branch $g(z)$. The other branches of the inverse $w(z)$ will be denoted by $g_{j}(z), j= \pm 1, \pm 2, \ldots$

The same figure shows the curves in the $w$-plane which correspond to circles $|z|=r$ in the $z$-plane. These curves are given by the equation

$$
u^{2}+v^{2}=r^{2} e^{2 u} .
$$

For $r<e^{-1}$ the curve consists of two parts, a small closed curve around the origin and a paraboloidal branch. For $r=e^{-1}$ the curve consists of a single track with a node at $w=1$. For $r>e^{-1}$ we obtain a single simple curve. We note that $d z / d w=(1-w) e^{-w}$ so that $z=e^{-1}, w=1$ gives a branchpoint of order two.

The branches $f(z), g(z)$ and $g_{j}(z), j= \pm 1, \pm 2, \ldots$, correspond to the various sheets of a Riemannian plane. The sheet of the main branch $f(z)$ has a cut at the positive real axis $e^{-1}<x<\infty$.

The sheet of $g(z)$ has the same cut but also a cut at the negative real axis $-\infty<x<0$. These sheets are connected at the cut $e^{-1}<x<\infty$. All other sheets have the single cut $-\infty<x<0$ and are connected to each other and to the second sheet in a screw-like fashion.

\section{The asymptotic expansion of $\boldsymbol{\Phi}_{n}^{+}(\lambda)$}

The functions $\varphi$ and $\psi$ determined by (1.4) and (1.5) may be continued analytically in the following way. We have for $|z|<e^{-1}$

$$
\begin{aligned}
\varphi(z, \alpha) & =\sum_{k=0}^{\infty}\left(\frac{(k+\alpha)^{k}}{k !}-\frac{(k+\alpha)^{k-1}}{(k-1) !}\right) z^{k} \\
& =\sum_{k=0}^{\infty} \frac{z^{k}}{2 \pi i} \int_{L} e^{w(k+\alpha)} w^{-k-1}(1-w) d w \\
& =\frac{1}{2 \pi i} \int_{L} e^{\alpha w} \frac{(1-w) e^{-w}}{w e^{-w}-z} d w
\end{aligned}
$$

where $L$ is a contour such that $\left|w e^{-w}\right|>|z|$. 
In view of the discussion of the preceding section, we may take for $L$ the vertical line $\operatorname{Re} w=1$. Since $e^{\alpha w}$ tends to zero on the left of $L$ the integral last obtained equals the residue at the only pole of $w=f(z)$, i.e.

$$
\varphi(z, \alpha)=\exp \alpha f(z)=z^{-\alpha} f^{\alpha}(z) \text {. }
$$

We note in passing that from $\partial \varphi / \partial \alpha \rightarrow f(z)$ for $\alpha \rightarrow 0$ the result (2.9) follows.

The expression (3.2) determines the complete analytical behaviour of $\varphi(z, \alpha)$. In particular $\varphi(z, \alpha)$ has the branch point $z=e^{-1}$ as the only singularity.

In a similar way we have

$$
\psi(z, \alpha)=\sum_{k=0}^{\infty} \frac{z^{k}}{2 \pi i} \int_{L} e^{w(k-\alpha)} w^{-k-1} d w=\frac{1}{2 \pi i} \int_{L} e^{-\alpha w} \frac{e^{-w}}{w e^{-w}-z} d w,
$$

where $L$ is the vertical line $\operatorname{Re} w=1$. We note that the terms with $k \leqq \alpha$ which are included here do not contribute to the sum.

The integral (3.3) now equals the sum of the residues $w=g(z)$ and $g_{j}(z)$, $j= \pm 1, \pm 2, \ldots$, which are situated on the right of $L$, i.e.

$$
\psi(z, \alpha)=\frac{e^{-\alpha g}}{g-1}+\sum_{j} \frac{e^{-\alpha g_{j}}}{g_{j}-1} .
$$

Substitution of these results in (1.8) gives

$$
S_{n}(\alpha)=\frac{1}{2 \pi i} \oint \frac{e^{-\alpha(g-f)}}{g-1} z^{-n-1} d z+R,
$$

where the remainder contains similar terms with $g_{j}$ instead of $g$. However, the behaviour of $f, g$ and $g_{j}$ at $z=e^{-1}$ shows that $R$ is asymptotically negligible. In fact, a slightly more accurate estimate shows that

$$
R=n^{-3 / 2} e^{n} O\left(e^{-1.6 \alpha}\right) .
$$

What we have found so far is important enough to state as a separate lemma.

\section{Lemma 3.}

$$
\Phi_{n}^{+}(\lambda)=1-\frac{n ! n^{-n}}{2 \pi i} \oint \frac{e^{-\alpha(g-f)}}{g-1} z^{-n-1} d z+O\left(e^{-1.6 \lambda \sqrt{n}}\right) .
$$

The asymptotically significant part on the right-hand side of (3.5) may be transformed into

$$
\frac{1}{2 \pi i} \int_{-i \infty}^{i \infty} z^{-n} e^{-2 \alpha p}(\ln g)^{\prime} d p
$$

by using the substitution (2.5).

Writing

$$
-\ln z=1+\frac{1}{2} p^{2}+B(p), \quad d \ln g / d p=A(p)
$$

it follows in view of (2.8) and (2.9) that $A(p)$ and $B(p)$ are given analytically by (1.11) and that

$$
A(p)=1-\sum_{k=1}^{\infty} \frac{B_{2 k}}{(2 k) !}(2 p)^{2 k-1},
$$


and

$$
B(p)=\sum_{k=2}^{\infty} \frac{(2 k+1) B_{2 k}}{2 k(2 k) !}(2 p)^{2 k}
$$

Then (3.7) may be written as

$$
\frac{e^{+n}}{2 \pi i} \int_{-i \infty}^{i \infty} e^{1 / 2 n p^{2}-2 \alpha p} A(p) \exp \{n B(p)\} d p .
$$

Hence neglecting the order term on the right-hand side of (3.7) the following asymptotic equality is obtained

$$
\Phi_{n}^{+}(\lambda) \approx 1-\frac{n !}{n^{n+1 / 2} e^{-n}} \frac{1}{2 \pi i} \int_{-i \infty}^{i \infty} e^{1 / 2 t^{a}-2 \lambda t} A\left(t n^{-1 / 2}\right) \exp \left\{n B\left(t n^{-1 / 2}\right)\right\} d t
$$

The result (1.9) of the theorem stated in the introduction now follows easily by observing that for integer $m$

$$
\int_{-i \infty}^{i \infty} e^{1 / 2 t^{2}-2 \lambda t} t^{m} d t=i \sqrt{2 \pi} e^{-2 \lambda^{2}} \mathrm{He}_{m}(2 \lambda),
$$

where $\mathrm{He}_{m}$ denotes the $m^{t h}$ polynomial of Hermite, and that by the expansion

$$
A\left(t n^{-1 / 2}\right) \exp \left\{n B\left(t n^{-1 / 2}\right)\right\}=\sum_{j=0}^{\infty} f_{j}(t) n^{-1 / 2 j}
$$

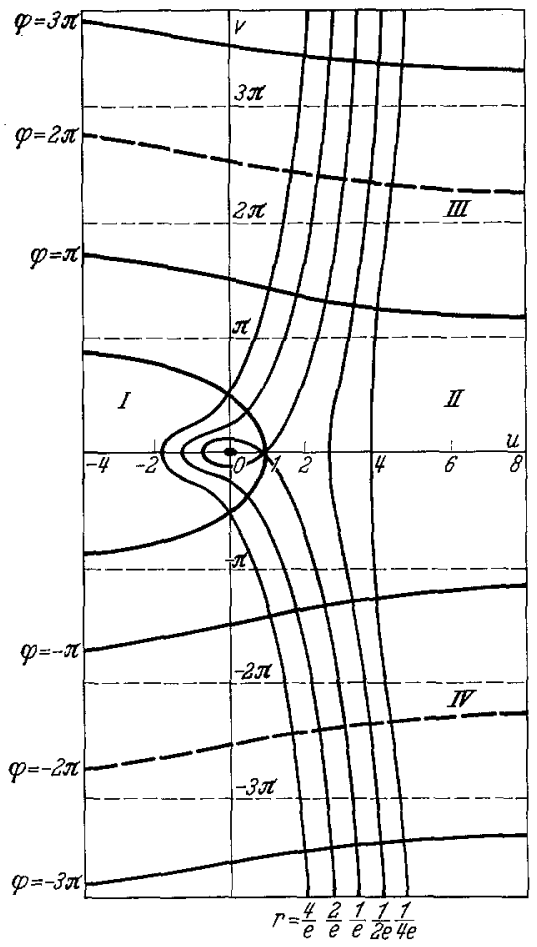

Fig. 1. Conformal mapping $z=w e^{-w}, z=r e^{i \varphi}$, $w=u+i v$. a set of polynomials $f_{j}(t)$ is obtained. We note that the latter expansion converges only for $|t|<1 / 2 \pi n^{1 / 2}$. Therefore the expansion (1.9) which is obtained by integration over an infinite $t$-interval is asymptotic by nature. The polynomials $f_{j}(t)$ can easily be calculated explicitly by using the series expansions (3.10) and (3.11). The first few polynomials are given in the introduction.

If the expansion (1.9) is combined with the well-known Stirling expansion

$$
\frac{n !}{n^{n} e^{-n} \sqrt{2 \pi n}}=1+\frac{1}{12 n}+\frac{1}{288 n^{2}}+\cdots
$$

the corollary (1.12) is obtained.

\section{Appendix}

Starting from the expression (1.8) we note that there exists a circle $|z|=R>e^{-1}$ in the interior of which $\varphi$ and $\psi$ have the only singularity $z=e^{-1}$. Then the path of integration may be deformed into two straight parts at the upper and lower side 
of the slit at the positive real axis from $z=e^{-1}$ to $z=R$ and a circular part along the circle $|z|=R$. Since we are interested only in asymptotic expressions the contribution from the circular part may be neglected and the straight parts may be formally extended to infinity. Since by (3.2) and (3.4)

$$
\left\{\begin{array}{l}
\varphi(z, \alpha)=\exp \alpha f(z) \\
\psi(z, \alpha)=\frac{\exp -\alpha g(z)}{g-1}+H(z)
\end{array}\right.
$$

where $f(z)$ and $g(z)$ are defined in the first paragraph of section 2 and where $H(z)$ is holomorphic at the positive real axis (1.8) changes into the asymptotic equality

$$
S_{n}(\alpha) \approx \frac{1}{2 \pi i} \int^{1^{+}} \frac{\exp \alpha(f-g)}{g-1} z^{-n-1} d z
$$

The behaviour of $f(z)$ and $g(z)$ at the branch point $z=e^{-1}$ is given by (2.4). Putting

$$
s=\sqrt{2-2 e z}
$$

where $s$ takes a positive real value for real $z$ with $z<e^{-1}$, we have

$$
\begin{aligned}
& f-g=-2 s+O\left(s^{3}\right) \\
& g-1=\quad s+\frac{1}{3} s^{2}+O\left(s^{3}\right) .
\end{aligned}
$$

Substitution in (4.2) gives

$$
S_{n}(\alpha) \approx \frac{e^{n}}{2} \frac{\pi i}{i \infty} \int_{-i \infty}^{\infty}\left(1-\frac{1}{2} s^{2}\right)^{-n-1} e^{-2 \alpha s}\left(1-\frac{1}{3} s+\cdots\right) d s .
$$

Putting $s=t / \sqrt{n}$ we obtain for $n \rightarrow \infty$ in view of $(1.7)$

$$
\begin{aligned}
\Phi_{n}^{+}(\lambda) & \approx 1-\frac{n !}{n^{n+1 / 2} e^{-n}} \frac{1}{2 \pi i} \int_{-i \infty}^{i \infty}\left(1-\frac{t^{2}}{2 n}\right)^{-n-1} e^{-2 \lambda t}\left(1-\frac{1}{3} \frac{t}{\sqrt{n}}+\cdots\right) d t \\
& =1-\frac{1}{i \sqrt{2 \pi}} \int_{-i \infty}^{i \infty} e^{1 / 2 t^{2}-2 \lambda t}\left(1-\frac{1}{3} \frac{t}{\sqrt{n}}\right) d t+O\left(n^{-1}\right) .
\end{aligned}
$$

Finally by shifting the path of integration to $(2 \lambda-i \infty, 2 \lambda+i \infty)$ it follows by changing $t$ into the real variable $u$ by the substitution $t=2 \lambda+i u$

$$
\Phi_{n}^{+}(\lambda)=1-\frac{e^{-2 \lambda^{2}}}{\sqrt{2 \pi}} \int_{-\infty}^{\infty} e^{-1 / 2 u^{2}}\left\{1-\frac{2 \lambda+i u}{3 \sqrt{n}}\right\} d u+O\left(n^{-1}\right)
$$

so that

$$
\Phi_{n}^{+}(\lambda)=1-e^{-2 \lambda^{2}}\left(1-\frac{2 \lambda}{3 \sqrt{n}}+O\left(n^{-1}\right)\right)
$$

which is SmrRnov's original result. 


\section{References}

[1] Smirnov, N. V.: Uspechi mat. Nauk. 10, 179-206 (1944).

[2] Tsias, Chan Li: Shuxue Jinzhan $1,775-790$ (1955) (Chinese).

[3] Birnadum, Z. W., and F. H. TingeY : Ann. math. Statistica 22, 592-596 (1951).

[4] Gredenko, B. V.: Tagung in Berlin Wahrscheinlichkeitsrechnung und Math. Stat. Deutscher Verl. d. Wiss. 97-107 (1954).

[5] - V.S. KorolUk, and A. V. Skorokнod: Proc. fourth Berkeley Symp. on mathematical statistics and probability, Berkeley, Univ. of Calif., 153-170 (1961).

\section{Mathematisch Centrum \\ $2^{\text {de }}$ Boerhaavestr. 49 \\ Amsterdam $\mathrm{O}$}

(Received July 28, 1962) 